\title{
Nivel de actividad física, equilibrio energético y exceso de peso en jóvenes universitarios
}

\section{Physical activity level, energy balance and excess weight in university students}

\author{
Eduardo Alberto Gómez Infante ${ }^{1}$, Oswaldo Ceballos Gurrola² y María Cristina Enríquez Reyna ${ }^{2 *}$ \\ 1 Unidad Académica Hermosillo, Universidad Estatal de Sonora, Hermosillo, Sonora (México). \\ 2 Facultad de Organización Deportiva, Universidad Autónoma de Nuevo León, Nuevo León (México).
}

\begin{abstract}
Resumen: La asociación entre la nutrición, actividad física y exceso de peso han sido estudiadas como determinantes del sobrepeso y enfermedades crónicas durante la adultez. Poco se sabe de la población universitaria en lugares donde convergen circunstancias climáticas, nutricionales y culturales adversas para la salud. Se analizaron las diferencias entre el nivel de actividad física, el equilibrio energético y la grasa corporal considerando la prevalencia de exceso de peso. Se aplicaron el International Physical Activity Questionnaire, el Recordatorio de 24 horas de pasos múltiples, análisis de bioimpedancia y mediciones antropométricas. De los 369 participantes, el $44.2 \%$ de los hombres y $23.2 \%$ de las mujeres presentó exceso de peso de acuerdo al IMC (>25 kg/m2). Se encontraron diferencias entre grupos al respecto del nivel de actividad y la grasa corporal $(p<.01)$. El nivel de actividad se asoció de forma inversa con el equilibrio energético y la grasa corporal.

Palabras clave: Sobrepeso, actividad física, equilibrio energético, índice de masa corporal, estudiantes.
\end{abstract}

Abstract: The association between nutrition, physical activity and excess weight have been studied as determinants of overweight and chronic diseases during adulthood. Little is known about the university population where climatic, nutritional and cultural circumstances are adverse for health. We analyzed the differences between the physical activity level, energy balance and body fat considering excess weight. The International Physical Activity Questionnaire, the 24-hour multi-step reminder, bioimpedance analysis and anthropometric measurements were applied. Three hundred sixty nine participated of which $44.2 \%$ of men and $23.2 \%$ of women had excess weight according to BMI (>25 kg/ m2). Differences were found between groups regarding the level of activity and body fat $(\mathrm{p}<.01)$. The level of activity was inversely associated with energy balance and body fat. Key words: Overweight, physical activity, energy expenditure, body mass index, students.

\section{Introducción}

A nivel mundial, el cambio en los estilos de vida ha dado lugar a una creciente obesidad que es considerada factor de riesgo para aparición de enfermedades no transmisibles (ENT). Las ENT son causa del $60 \%$ de la mortalidad y el $45 \%$ de la morbilidad, mientras que en México se asocian con el 78\% de las defunciones totales (Morales, Godoy, López, \& Alonso, 2016). En el 2016, la prevalencia combinada de sobrepeso y obesidad fue del $72.5 \%$ en mexicanos de 20 años y más (Shamah-Levy, Cuevas-Nasu, Rivera-Dommarco, \& Hernández-Ávila, 2016), se considera que es un problema de salud pública que requiere acciones multisectoriales para su control (Lorenzini et al., 2015; Morales et al., 2016). El aprovechamiento nacional del bono demográfico por el que el país transita, depende en gran parte de la salud de la población joven.

En ocasiones la etapa universitaria predispone a la adquisición de estilos de vida inapropiados que posteriormente podrían repercutir en la salud. A pesar del nivel educativo, la población universitaria, se considera un colectivo vulnerable

Dirección para correspondencia [Correspodence address]: María Cristina Enríquez Reyna. Universidad Autónoma de Nuevo León, Facultad de Organización Deportiva, Cd. Universitaria, s/n, C.P. 66455. San Nicolás de los Garza, Nuevo León (México).

E-mail: maria.enriquezryn@uanl.edu.mx desde el punto de vista alimentario ya que comienza a responsabilizarse de su alimentación, atraviesa un período crítico en la consolidación de hábitos y conductas importantes para la salud futura (Sánchez-Socarrás \& Aguilar-Martínez, 2015). La situación de la juventud en una región fronteriza con clima desértico y creencias culturales características de Sonora, México (Calvario \& Díaz, 2017) retoma especial interés debido al cambio necesario en la cultura física hacia la educación y el cuidado de la salud (Angelucci, Cañoto, \& Hernández, 2017).

El equilibrio energético (EE) resultante de la alimentación, refleja el equilibrio entre el consumo y gasto calórico resultante de la actividad física (AF); cualquier desequilibrio, puede aumentar o disminuir la grasa y el peso corporal. En un EE positivo, se acumula grasa y peso corporal mientras que en uno negativo, se pierden. Algunos estudios han demostrado asociación entre el comportamiento sedentario y el sobrepeso en estudiantes universitarios, donde la inactividad física como estilo de vida es un factor determinante de enfermedades crónicas (López, 2016; Rangel-Caballero, Rojas-Sánchez, \& Gamboa-Delgado, 2014). La dieta moderna resulta ser abundante en grasas y poco variada; mientras que la AF puede crear un déficit de energía a través de un mayor gasto calórico. Por esta razón, la AF y el ejercicio tienen potencial como 
parte de la solución para la epidemia de la obesidad en la que prevalece una EE positivo para el cuerpo (Wiklund, 2016).

La etapa universitaria debiera fungir como una excelente oportunidad para la educación y el desarrollo de buenos hábitos de alimentación y de AF. La descripción de la relación entre el nivel de AF, EE y la grasa corporal (GC) en consideración a la presencia de exceso de peso ayudará a reconocer la influencia entre estas variables y fortalecerá la promoción de la cultura física y nutricional entre la población joven. No se encontraron reportes descriptivos del EE en jóvenes mexicanos, esto puede deberse a la complejidad de su medición que generalmente se realiza para el análisis personal y no grupal. El análisis de las características del EE en relación a la tendencia hacia la obesidad entre el colectivo de jóvenes universitarios, será de utilidad para determinar estrategias que permitan el diseño de intervenciones educativas, físicas y/o nutricionales a favor de su salud. En base a lo anterior, se propuso analizar las diferencias en el nivel de AF, el EE y la GC considerando la prevalencia de exceso de peso en jóvenes del campus de la salud de una universidad pública del noroeste de México. Además, se revisó la asociación entre el nivel de AF y la presencia de exceso de peso a partir del índice de masa corporal (IMC).

\section{Material y métodos}

Estudio descriptivo-transversal y correlacional en una población de 797 estudiantes adscritos al campus del área de la salud de una institución universitaria pública. A partir del listado de estudiantes inscritos se estimó el tamaño de muestra necesario considerando un nivel de confianza del 95\% y límite de error estimado del 5\%, quedando una muestra final de 369. Se aplicó un muestreo por estratos a partir del sexo y semestre. Se incluyó a estudiantes inscritos que aceptaron participar voluntariamente en el estudio. Se excluyó a mayores de 30 ańos y a quienes declararon ser atletas de alto rendimiento. Se eliminaron los datos de participantes que no completaron las pruebas y mediciones. Se aplicó la firma del consentimiento informado para el cumplimiento de lo expuesto en la Declaración de Helsinki.

\section{Actividad física}

Se midió con el International Physical Activity Questionnaire con validez reconocida para determinar el nivel de AF en METs de acuerdo a la intensidad (baja, moderada o vigorosa), frecuencia (días por semana) y duración de la AF (Medina, Barquera, \& Janssen, 2013). Se dividió la muestra en tertiles resultando los siguientes puntos de corte específicos: el nivel de AF se clasificó como bajo cuando el participante reportó actividad menor de 492 METs; moderado, de 492 a 2294 METs; y alto con actividad superior a 2294 METs. Los valores determinados resultan ser inferiores a los reportados por los autores del cuestionario (International Physical Activity Questionnaire, s. f.).

\section{Equilibrio energético}

Es el principal factor determinante del peso corporal y del tamańo de los depósitos de tejido adiposo en adultos; se refiere a la diferencia entre el consumo calórico y el gasto energético total. El EE equivale al mantenimiento del peso corporal; cuando el EE es positivo, se gana peso y cuando es negativo, se pierde. Para estimar el consumo calórico se aplicó el Cuestionario de Recordatorio de 24 Horas de Pasos Múltiples. Este método consiste en interrogar al sujeto sobre todo lo que ingirió el día anterior: sólidos y líquidos (Haua, 2010). Es una técnica rápida de administrar ya que generalmente lleva entre 20 y 30 minutos, en el cual el entrevistador pregunta el tipo, modo de preparación, nombre comercial, ingredientes de la receta y otras características; éste permite recordar detalles sobre los alimentos y bebidas consumidas en diversas ocasiones y reduce el riesgo de omitir alguno por olvido. Para minimizar los errores en la estimación de las porciones, se usaron modelos de alimentos, los cuales pueden ser fotografías de alimentos o réplicas de alimentos, que son susceptibles de ayudar a estimar el tamaño de las porciones (Suverza, 2010). Para este estudio, la conversión de los datos sobre la ingesta de alimentos a cantidad de kilocalorías y nutrimentos consumidos, implicó recurrir a la Tablas de Composición de Alimentos (Muñoz, 2010). Dichas tablas incluyen un listado de alimentos, además del contenido de energía y de 25 nutrimentos por cada 100 gramos de alimento. De modo que, para conocer el contenido nutrimental de una porción específica, se debió construir una ecuación en el programa Excel para obtener la cantidad estimada de dichos nutrimentos en calorías y unidades métricas de los nutrimentos cuantificados.

Se realizó análisis de bioimpedancia electromagnética utilizando el Body Scan Plus II* (Jawson Medical. Korea 2008) que utiliza micro-corriente y múltiples frecuencias entre 1 $\mathrm{kHz}$ y $1000 \mathrm{kHz}$ para determinar el gasto energético total (sumatoria de energía consumida por el cuerpo). Para la medición se tomaron las precauciones recomendadas al respecto del tiempo de medición (por las mañana), ayuno ( 4 horas), reposo (4 horas), consumo de alcohol y otros alimentos, vaciamiento de vejiga, uso de ropa ligera y temperatura de la zona de medición.

\section{Mediciones antropométricas}

Se registraron la edad y características antropométricas para la descripción de los participantes. La medición de la estatura, peso (Báscula SECA modelo 700 con estadiómetro integrado), circunferencia de cintura y la sumatoria de cuatro pliegues (tricipital, bicipital, subescapular y suprailíaco) se realizó por 
duplicado siguiendo las normas internacionales para la evaluación antropométrica para cada paso del protocolo de medición. Se calculó el IMC dividiendo el peso de la persona $(\mathrm{kg})$ entre el cuadrado de su talla en metros $\left(\mathrm{m}^{2}\right)$. Se siguió el criterio de la Organización Mundial de la Salud para determinar exceso de peso cuando se tuviera un IMC mayor a $25 \mathrm{~kg} / \mathrm{m}^{2}$.

Para la estimación del porcentaje de GC se realizó la medición y suma de cuatro pliegues con cinta antropométrica marca Lufkin Executive Thinline modelo W606 PM de acero flexible y calibrador marca Slim Guide de acuerdo a los parámetros del ISAK (Albarran \& Holway, 2005). Se usó la ecuación de Siri (para los mayores de 18 años por plicometría) cuya expresión matemática es: \%GC $=[(4.95 /$ densidad $)-4.5]$ x 100. La densidad corporal (D) se obtuvo mediante la ecuación de Durnin y Womersley: Densidad = C $-\mathrm{M} x \log 10 \sum$ cuatro pliegues. Para esta ecuación se requieren de los coeficientes $\mathrm{C}$ y $\mathrm{M}$ que son obtenidos de la suma de los cuatro pliegues reflejados en las tablas ya conocidas de manera específica para cada grupo de edad en cada sexo (Durnin \& Womersley, 1974).

\section{Análisis estadístico}

Los datos se analizaron mediante el programa Statistical Package for Social Science (SPSS) versión 22.0 . Para el análisis descriptivo con valor promedio y desviación estándar se presentan los datos de la muestra completa, por sexo y considerando la presencia o ausencia de exceso de peso. Posteriormente, a partir de la división de la muestra en tertiles de AF en METs, se describe la frecuencia y datos del nivel de AF, EE y GC por estrato (nivel de AF bajo, moderado o alto). Se consideró un valor crítico para el alfa de .05. Para el análisis de las diferencias por estratos se aplicaron pruebas $t$ para muestras independientes y análisis de varianza. Se corrieron pruebas de razón de momios para determinar el riesgo de exceso de peso por nivel de AF.

\section{Resultados}

Los resultados muestran una participación de 369 estudiantes del área de la salud, la mayoría (76.7\%) del sexo femenino. El $44.2 \%$ de los hombres y $23.2 \%$ de las mujeres presentó exceso de peso de acuerdo al IMC. La tabla 1, muestra los valores y diferencias a partir de los puntos de corte en tertiles para el nivel de AF, EE y GC para la muestra completa y por sexo de acuerdo a la presencia o ausencia de exceso de peso.

Tabla 1. Diferencias en el nivel de actividad física, el equilibrio energético y la grasa corporal en la muestra completa, por sexo y de acuerdo a la presencia o ausencia de exceso de peso.

\begin{tabular}{|c|c|c|c|c|c|c|c|c|c|c|}
\hline \multirow[b]{2}{*}{ Variables } & \multirow[b]{2}{*}{ Muestra completa } & \multicolumn{5}{|c|}{ Hombres } & \multicolumn{3}{|c|}{ Mujeres } & \multirow{2}{*}{$\begin{array}{c}\mathrm{H} / \mathrm{M} \\
P\end{array}$} \\
\hline & & Todos $(H)$ & Con EP & $\operatorname{Sin} E P$ & $p$ & Todas (M) & Con EP & $\operatorname{Sin} E P$ & $p$ & \\
\hline AF, METs & $\begin{array}{c}1956.02 \pm 1974.76 \\
(n=369)\end{array}$ & $\begin{array}{c}2783.02 \pm 2243.22 \\
(n=86)\end{array}$ & $\begin{array}{c}2560.67 \pm 2149.28 \\
(n=38)\end{array}$ & $\begin{array}{c}2959.04 \pm 2322.13 \\
(n=48)\end{array}$ & & $\begin{array}{c}1704.71 \pm 1816.66 \\
(n=283)\end{array}$ & $\begin{array}{c}1779.67 \pm 1681.91 \\
(n=66)\end{array}$ & $\begin{array}{c}1681.91 \pm 1773.52 \\
(n=217)\end{array}$ & & $* *$ \\
\hline $\begin{array}{l}\text { N Bajo, }<492 \\
\text { METs de AF }\end{array}$ & $\begin{array}{c}351.86 \pm 91.59 \\
(n=123)\end{array}$ & $\begin{array}{c}397.14 \pm 96.75 \\
(n=14)\end{array}$ & $\begin{array}{c}409.19 \pm 52.21 \\
\quad(n=8)\end{array}$ & $\begin{array}{c}381.08 \pm 141.35 \\
\quad(n=6)\end{array}$ & & $\begin{array}{c}346.04 \pm 89.72 \\
(n=109)\end{array}$ & $\begin{array}{c}332.25 \pm 96.48 \\
\quad(n=28)\end{array}$ & $\begin{array}{c}350.81 \pm 87.38 \\
\quad(n=81)\end{array}$ & & * \\
\hline $\begin{array}{l}\text { N Moderado, } \\
492-2294 \\
\text { METs de AF }\end{array}$ & $\begin{array}{c}1211.43 \pm 529.81 \\
(n=123)\end{array}$ & $\begin{array}{c}1282.19 \pm 540.14 \\
(n=29)\end{array}$ & $\begin{array}{c}1277.79 \pm 529.00 \\
(n=12)\end{array}$ & $\begin{array}{c}1285.29 \pm 564.05 \\
(n=17)\end{array}$ & & $\begin{array}{c}1189.60 \pm 527.58 \\
(n=94)\end{array}$ & $\begin{array}{c}1306.16 \pm 555.12 \\
(n=19)\end{array}$ & $\begin{array}{c}1160.07 \pm 520.08 \\
\quad(n=75)\end{array}$ & & \\
\hline $\begin{array}{l}\text { N Vigoroso, } \\
>2294 \text { METs } \\
\text { de AF }\end{array}$ & $\begin{array}{c}4304.79 \pm 1660.89 \\
(n=123)\end{array}$ & $\begin{array}{c}4572.00 \pm 1804.72 \\
(n=43)\end{array}$ & $\begin{array}{c}4372.14 \pm 1748.52 \\
\quad(n=18)\end{array}$ & $\begin{array}{c}4715.90 \pm 1866.19 \\
(n=25)\end{array}$ & & $\begin{array}{c}4161.16 \pm 1571.21 \\
(n=80)\end{array}$ & $\begin{array}{c}4386.22 \pm 1715.59 \\
\quad(n=19)\end{array}$ & $\begin{array}{c}4091.06 \pm 1531.81 \\
\quad(n=61)\end{array}$ & & \\
\hline EE, calorías & $312.29 \pm 430.06$ & $429.89 \pm 511.74$ & $393.29 \pm 498.34$ & $458.87 \pm 525.51$ & & $276.55 \pm 396.12$ & $287.19 \pm 406.46$ & $273.31 \pm 393.83$ & & * \\
\hline $\begin{array}{l}\text { EE-N Bajo, } \\
\text { calorías }\end{array}$ & $364.54 \pm 424.22$ & $627.63 \pm 626.67$ & $654.66 \pm 817.52$ & $591.58 \pm 287.48$ & & $330.75 \pm 381.96$ & $286.44 \pm 393.17$ & $346.06 \pm 379.29$ & & * \\
\hline $\begin{array}{l}\text { EE-N Mode- } \\
\text { rado, calorías }\end{array}$ & $309.37 \pm 444.60$ & $349.28 \pm 400.76$ & $343.21 \pm 368.02$ & $357.88 \pm 460.08$ & & $297.06 \pm 458.59$ & $367.01 \pm 426.19$ & $279.34 \pm 467.48$ & & \\
\hline $\begin{array}{l}\text { EE-N Alto, } \\
\text { calorías }\end{array}$ & $262.95 \pm 418.36$ & $419.89 \pm 532.68$ & $300.74 \pm 293.69$ & $505.67 \pm 645.868$ & & $178.59 \pm 314.37$ & $208.46 \pm 412.08$ & $169.29 \pm 280.740$ & & $* *$ \\
\hline GC, $\%$ & $26.78 \pm 6.65$ & $19.12 \pm 5.99$ & $23.33 \pm 5.62$ & $15.78 \pm 3.77$ & $* *$ & $29.11 \pm 4.85$ & $32.76 \pm 4.54$ & $28.01 \pm 4.38$ & $* *$ & $* *$ \\
\hline GC-N Bajo, \% & $29.16 \pm 5.60$ & $22.23 \pm 7.58$ & $26.48 \pm 6.01$ & $16.57 \pm 5.61$ & $* *$ & $30.05 \pm 4.64$ & $34.40 \pm 3.76$ & $28.55 \pm 3.92$ & $* *$ & $* *$ \\
\hline $\begin{array}{l}\text { GC-N Mode- } \\
\text { rado, } \%\end{array}$ & $26.52 \pm 6.14$ & $20.41 \pm 6.04$ & $26.04 \pm 3.87$ & $16.43 \pm 3.60$ & ** & $28.40 \pm 4.83$ & $31.64 \pm 4.97$ & $27.57 \pm 4.46$ & $* *$ & $* *$ \\
\hline GC-N Alto, $\%$ & $24.68 \pm 7.35$ & $17.23 \pm 4.74$ & $20.12 \pm 4.85$ & $15.15 \pm 3.43$ & ** & $28.69 \pm 5.00$ & $31.47 \pm 4.61$ & $27.82 \pm 4.84$ & $* *$ & $* *$ \\
\hline
\end{tabular}

$\mathrm{EP}=$ Exceso de Peso; H= Hombres; M= Mujeres; AF= Actividad Física; METs = unidades de gasto energético; $\mathrm{N}=\mathrm{Nivel} ; \mathrm{EE}=\mathrm{Equilibrio} \mathrm{energético;} \mathrm{GC} \mathrm{=} \mathrm{Grasa} \mathrm{Corporal.}$ ${ }^{*} p<.05 ;{ }^{* *} p<.01$ 
En la figura 1 se representan las diferencias en el EE, GC y el IMC por nivel de AF. Se observaron diferencias entre grupos al respecto del nivel de AF y la GC (F2,366 $=15.215, \mathrm{p}=.000$,
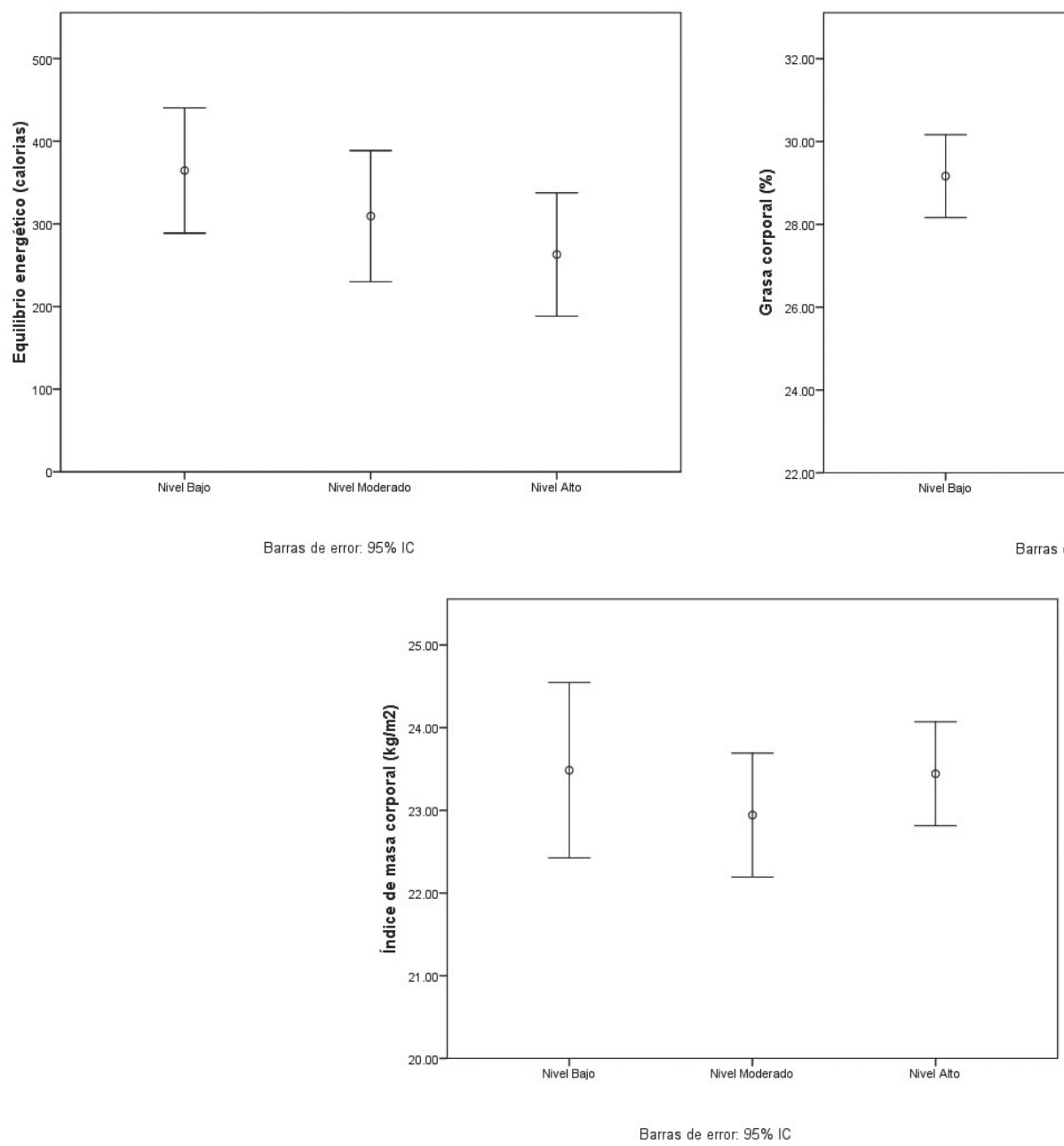

Figura 1. Diferencias en el equilibrio energético, la grasa corporal y el índice de masa corporal por nivel de actividad física en jóvenes universitarios.

En la tabla 2 se presenta la descripción de datos antropométricos y consumo calórico en el total de la muestra.
Etha2 $=.077)$. Con tendencia sin significancia en relación al EE y el IMC $(p>.05)$.

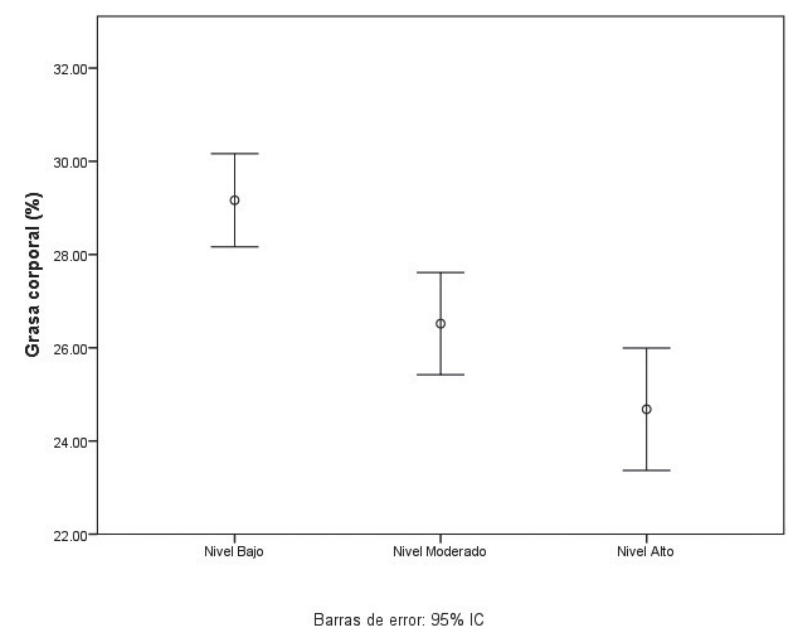

Aarras de error: $95 \%$ IC 
Tabla 2. Edad, características antropométricas y de consumo de los participantes estratificados por sexo y de acuerdo a la presencia o ausencia de exceso de peso.

\begin{tabular}{|c|c|c|c|c|c|c|c|c|c|c|}
\hline & \multirow[b]{2}{*}{$\begin{array}{c}\text { Todos } \\
(n=369)\end{array}$} & \multicolumn{5}{|c|}{ Hombres } & \multicolumn{2}{|c|}{ Mujeres } & \multicolumn{2}{|c|}{$\mathrm{H} / \mathrm{M}$} \\
\hline & & $\begin{array}{l}\text { Todos H } \\
(n=86)\end{array}$ & $\begin{array}{l}\text { Con EP } \\
(n=38)\end{array}$ & $\begin{array}{c}\text { Sin EP } \\
(n=48)\end{array}$ & $p$ & $\begin{array}{l}\text { Todas } \mathrm{M} \\
(n=283)\end{array}$ & $\begin{array}{l}\text { Con EP } \\
(n=66)\end{array}$ & $\begin{array}{c}\text { Sin EP } \\
(n=217)\end{array}$ & $p$ & $p$ \\
\hline Edad (años) & $20.17 \pm 1.91$ & $20.34 \pm 2.31$ & $20.45 \pm 2.67$ & $20.25 \pm 2.05$ & & $20.12 \pm 1.77$ & $19.88 \pm 1.68$ & $20.20 \pm 1.80$ & & \\
\hline Estatura (m) & $1.65 \pm 0.09$ & $1.75 \pm 0.07$ & $1.76 \pm 0.08$ & $1.75 \pm 0.07$ & & $1.62 \pm 0.06$ & $1.62 \pm .06$ & $1.62 \pm .06$ & & $* *$ \\
\hline Peso $(\mathrm{kg})$ & $63.88 \pm 15.26$ & $77.32 \pm 16.81$ & $89.71 \pm 16.40$ & $67.51 \pm 8.79$ & $* *$ & $59.80 \pm 12.13$ & $75.47 \pm 12.14$ & $55.03 \pm 7.05$ & $* *$ & $* *$ \\
\hline $\operatorname{IMC}\left(\mathrm{kg} / \mathrm{m}^{2}\right)$ & $23.29 \pm 4.66$ & $25.09 \pm 5.54$ & $29.02 \pm 5.99$ & $21.98 \pm 2.18$ & $* *$ & $22.74 \pm 4.22$ & $28.70 \pm 3.71$ & $20.93 \pm 2.23$ & $* *$ & $* *$ \\
\hline $\mathrm{CC}, \mathrm{cm}$ & $77.32 \pm 11.01$ & $84.67 \pm 11.96$ & $93.76 \pm 11.25$ & $77.49 \pm 6.28$ & $* *$ & $75.08 \pm 9.68$ & $87.44 \pm 8.35$ & $71.32 \pm 6.37$ & $* *$ & $* *$ \\
\hline $\mathrm{S} 4 \mathrm{PC}(\mathrm{mm})$ & $63.95 \pm 23.58$ & $54.32 \pm 26.28$ & $71.76 \pm 28.29$ & $40.51 \pm 16.36$ & $* *$ & $66.87 \pm 21.92$ & $89.73 \pm 21.90$ & $59.91 \pm 16.57$ & $* *$ & $* *$ \\
\hline GC (\%) & $26.79 \pm 6.65$ & $19.12 \pm 5.99$ & $23.33 \pm 5.62$ & $15.78 \pm 3.77$ & $* *$ & $29.12 \pm 4.85$ & $32.77 \pm 4.54$ & $28.01 \pm 4.38$ & $* *$ & ** \\
\hline CCal (cal) & $2475.06 \pm 546.10$ & $2637.25 \pm 644.09$ & $2528.08 \pm 615.05$ & $2723.68 \pm 659.69$ & & $2425.77 \pm 503.65$ & $2476.53 \pm 573.66$ & $2410.34 \pm 480.75$ & & ** \\
\hline $\mathrm{GE}$ (cal) & $2162.78 \pm 479.96$ & $2207.36 \pm 533.86$ & $2134.79 \pm 461.94$ & $2264.81 \pm 582.94$ & & $2149.23 \pm 462.52$ & $2189.34 \pm 523.82$ & $2137.03 \pm 442.80$ & & \\
\hline
\end{tabular}

H= Hombres; M= Mujeres; EP = Exceso de Peso; IMC = Índice de Masa Corporal; S4PC = Sumatoria de cuatro pliegues; GC = Grasa corporal; CCal= Consumo Calórico; $\mathrm{GE}=$ Gasto Energético. ${ }^{* *} p<.01$.

Se revisó la asociación entre el nivel de AF y la presencia de exceso de peso en base al IMC. Los datos confirman el incremento del riesgo ante un nivel de actividad bajo (Tabla $3)$.

Tabla 3. Riesgo de presentar exceso de peso de acuerdo al índice de masa corporal por nivel de actividad física y sexo.

\begin{tabular}{lcccccc}
\hline \multirow{2}{*}{ Nivel de Actividad } & \multicolumn{3}{c}{ Todos } & \multicolumn{2}{c}{ Hombres } & Mujeres \\
\cline { 2 - 6 } & OR & IC 95\% & OR & IC 95\% & IC 95\% \\
\hline Bajo (<492 METs) & 1.083 & $.671-1.748$ & 1.867 & $.587-5.940$ & 1.237 & $.706-2.167$ \\
Moderado (492-2294 METs) & .799 & $.489-1.304$ & .842 & $.341-2.079$ & .765 & $.419-1.397$ \\
Alto (>2294 METs) & 1.041 & $.714-1.852$ & .828 & $.353-1.941$ & 1.034 & $.562-1.902$ \\
\hline
\end{tabular}

\section{Discusión}

Se analizaron las diferencias entre el nivel de AF, EE, el porcentaje de GC y consumo calórico de acuerdo a la presencia de exceso de peso en jóvenes universitarios. Por medio del análisis de riesgo, se identificó la susceptibilidad de presentar exceso de peso por sexo y nivel de AF. Los hallazgos de estudio pueden resumirse con lo siguiente: Se observó una tendencia negativa entre el reporte de $\mathrm{AF}$ y los niveles de EE y porcentaje de GC. En contraste, el IMC en jóvenes no muestra una tendencia similar; en quienes presentan un nivel de AF bajo se observa mayor promedio de IMC, el promedio baja para el nivel de AF moderado sin embargo, se incrementa ante nivel de AF alto.

La tendencia negativa del nivel de AF con el EE y la GC evidencia la influencia del nivel de AF para el control del peso corporal y la GC de la misma forma que se ha seńalado en reportes previos de la literatura (Drenowatz et al., 2015; Kelley, Kellery, \& Pate, 2014). La AF incrementa el gasto energético lo que incide en la GC y el EE. De la misma forma puede explicarse la tendencia al incremento del IMC en jóvenes con bajo nivel de AF. La falta de tendencia inversa entre el nivel de AF y el IMC puede deberse a cuestiones relacionadas con la masa muscular. El músculo tiene mayor peso e inclusive los atletas buscarán mayor consumo proteico con el fin expedito de incrementar la masa muscular por lo que en esos casos, el indicador de obesidad ideal no es el IMC sino más bien, el porcentaje de GC.

Se entiende que la vida cotidiana del estudiante universitario puede implicar la necesidad de un gran aporte calórico. Durante esta etapa, el estrés (incremento de la presión académica, cambios en los grupos de apoyo social, independencia del hogar familiar, responsabilidad en decisiones en materia de manejo del tiempo, entre otros) y las modificaciones en los estilos de vida (cambios en los hábitos dietéticos, incremento de consumo de alcohol y disminución de la AF, p. e.) se conjuntan y constituyen como factores prioritarios en el aumento del peso corporal (Soto-Ruiz et al, 2015). Sin embargo, en estudiantes en formación del campus de la salud, socialmente se esperaría que aplicaran los conocimientos que están adquiriendo mediante el desarrollo de estilos de vida nutricional y de ejercicio saludables. 
Entre los participantes de esta muestra, se observó preferencia por alimentos con alto valor calórico, a libre deman$\mathrm{da}$, con descuido de los adecuados horarios para consumo de alimentos e inactividad física persistente de la misma forma que en otros reportes de la literatura al respecto de la alimentación de los jóvenes (Cardozo, Cuervo, \& Murcia, 2016; Ortega-González et al., 2017; Rangel-Caballero, Rojas-Sánchez, \& Gamboa-Delgado, 2015). La alta carga energética y el bajo consumo de alimentos no procesados o bien en su estado natural, tiende a incrementar la GC y desequilibrar el EE (López, 2016).

Los resultados brindan una panorámica sobre la magnitud del alto riesgo de desarrollo de ENT que enfrentan los universitarios en consonancia con otros reportes de la literatura (López, 2016; Lorenzini et al., 2015; Morales et al., 2016; Shamah-Levy et al., 2016). Se confirma entonces la situación que enfrentan los estudiantes universitarios. Su condición de estudiantes los posiciona como privilegiados en formación para integrarse profesionalmente a la sociedad, pero por otro lado, queda reflejada su vulnerabilidad ante las dificultades socioculturales que el papel de estudiante conlleva. Los universitarios debieran considerar entonces, que no sólo deben estudiar y prepararse para integrarse a la fuerza laboral, sino que deben aprender a utilizar los medios necesarios para adquirir hábitos de salud que afecten de manera positiva su vida personal, profesional y laboral (Lorenzini et al., 2015).

La prevalencia de exceso de peso $(28.47 \%)$ en esta muestra supera otros reportes de investigaciones realizadas en el contexto universitario (Cardozo et al., 2016; Dos Santos et al., 2017; Ortega et al., 2017; Rangel et al., 2015; Soto et al., 2015); pero contrasta con el reporte de un campus de la salud ubicado en España (Rizo-Baeza, González-Brauer, \& Cortés, 2014). Esto puede explicarse debido a que los universitarios del noroeste de México experimentan una marcada tendencia hacia la cultura alimentaria norteamericana o una combinación de ella con la cultura alimentaria regional, en la que con- vergen un consumo elevado de proteínas de origen animal y grasas saturadas, así como un consumo frecuente de hidratos de carbono refinados.

Casi el $40 \%$ de los universitarios no realiza la AF diaria recomendada; estos valores concuerdan con los reportes (Dos Santos, Castillo, De Araujo, \& Giménez, 2017; Soto et al., 2015) pero contrastan con un reporte nacional de dos estados del sur (Carrillo et al., 2017), uno de Brasil (Savegnago et al., 2014) y Argentina (Pérez, Lanío, Zelarayán, \& Márquez, 2014). Las diferencias con los estados del sur del país pudieran explicarse por la tipología de clima de esos contextos; algo similar pudiera pensarse acerca de los países sudamericanos. Sin embargo, las diferencias en la cultura física y nutricional pudieran estar actuando a favor de los estilos de vida de los universitarios. Sin una cultura física apropiada, una vez que el universitario concluye sus estudios y comienza la etapa adulta, se enfrenta a nuevas barreras para el ejercicio como una alta carga laboral, responsabilidades familiares y sociales que lo alejan aún más de la AF. Los niveles de estrés se incrementan y la cantidad de tiempo libre para practicar AF o ejercicio disminuye, lo que favorece el desarrollo o agravamiento del cuadro de obesidad y las ENT.

Las limitaciones de estudio están en relación a las cuestiones propias del diseño transversal. Se vigiló con alto escrutinio la selección de participantes y momentos para la eficiencia en las mediciones por lo que se vigiló prevenir, dentro de lo posible, el sesgo de medición. En esta ocasión, se revisó exclusivamente al campus de la salud, por lo que los resultados reflejan las características de esa tipología de estudiante. Futuras investigaciones deberían replicarse en muestras de mayor tamańo con muestreo representativo de diferentes campus universitarios. Dados los resultados relativos al IMC en jóvenes con nivel de AF alto, sería conveniente investigar como incide el nivel de AF sobre otros indicadores de riesgo cardiovascular con estimación de la GC con diferentes técnicas de medición y laboratorio.

\section{Referencias}

1. Albarran, M. A. \& Holway, F. (2005). Estándares Internacionales para la Valoración Antropométrica (ISAK Manual). Universidad de Puerto Rico: Sociedad Internacional para el avance de la Kinantropometría; 2005.

2. Angelucci, L. T., Cańoto, Y., \& Hernández, M. J. (2017). Influencia del estilo de vida, el sexo, la edad y el IMC sobre la salud física y psicológica en jóvenes universitarios. Avances en Psicología Latinoamericana, 35(3), 531-546. Doi: http://dx.doi.org/10.12804/revistas.urosario.edu. co/apl/a.4454

3. Cardozo, L. A., Cuervo, Y., \& Murcia, J. (2016). Porcentaje de grasa corporal y prevalencia de sobrepeso-obesidad en estudiantes universitarios de rendimiento deportivo de Bogotá, Colombia. Nutr. clín. diet. Hosp, 36(3), 68-75. DOI: 10.12873/363cardozo

4. Carrillo, C. M. S., Molina, S. F., Del Río Valdivia, J., Figueroa, J. A. G., López, S. H., \& Moreno, P. J. F. (2017). Actividad física e IMC de los universitarios de Veracruz y Colima. Revista Mexicana de Investigación en Cultura Física y Deporte, 3(3), 53-69.
5. Deforche, B., Van Dyck, D., Deliens, T., De Bourdeaudhuij, I. (2015) Changes in weight, physical activity, sedentary behaviour and dietary intake during the transition to higher education: a prospective study. Int J Behav Nutr Phys Act, 15, 12-16. https://doi.org/10.1186/s12966015-0173-9

6. Dos Santos, J. S., Castillo, E., De Araujo, A. G., \& Giménez, F. J. (2017). Relación entre actividad física, composición corporal e imagen corporal en estudiantes universitarios. SPORT TK-Revista EuroAmericana de Ciencias del Deporte, 6(2), 39-48.

7. Drenowatz, C., Hand, G. A., Shook, R. P., Jakicic, J. M., Hebert, J. R., Burgess, S., \& Blair, S. N. (2015). The association between different types of exercise and energy expenditure in young non overweight and overweight adults. Appl Physiol Nutr Metab, 40(3), 211-217. DOI: 10.1139/apnm-2014-0310.

8. Durnin, J. V. \& Womersley, J. V. G. A. (1974). Body fat assessed from total body density and its estimation from skinfold thickness: measu- 
rements on 481 men and women aged from 16 to 72 years. Br J Nutr, 32(1), 77-97. DOI: https://doi.org/10.1079/BJN19740060

9. Haua, K. Alimentación: estrategias de evaluación. El ABCD de la Evaluación del Estado de Nutrición. 1a ed. México. McGraw-Hill; 2010.

10. International Physical Activity Questionnaire (s. f.). Guidelines for Data Precessing and Analysis of the International Physical Activity Questionnaire. Short and Long Forms. Disponible en: http://www.institutferran.org/documentos/scoring_short_ipaq_april04.pdf

11. Kelley, G. A., Kelley, K. S., \& Pate, R. R. (2014). Effects of exercise on BMI z-score in overweight and obese children and adolescents: a systematic review with meta-analysis. BMC Pediatrics, 14:225. DOI: 10.1111/jebm.12228.

12. López, L. I. G. (2016). Actividad física habitual de los alumnos de octavo semestre de la Facultad de Educación Física. Revista Mexicana de Investigación en Cultura Física y Deporte, 1(1), 80-99. Disponible en: $<$ http://revista.ened.edu.mx/index.php/revistaconade/article/view/11>

13. Lorenzini, R., Betancur-Ancona, D. A., Chel-Guerrero, L. A., SeguraCampos, M. R., Castellanos-Ruelas, A. F. (2015). Estado nutricional en relación con el estilo de vida de estudiantes universitarios. Nutr Hosp, 32(1), 94-100. DOI:10.3305/nh.2015.32.1.8872

14. Medina, C., Barquera, S., \& Janssen, I. (2013). Validity and reliability of the International Physical Activity Questionnaire among adults in Mexico. Rev Panam Salud Publica, 34(1), 21-28.

15. Morales, M. V., Godoy, S. M., López, M. A. C., \& Alonso, L. G. E. (2016). Enfoque integral de la importancia de la dieta en las condiciones actuales de salud de la población mexicana/comprehensive approach of diet importance on health status of the mexican population. Biotecnia, $18(1), 22-31$.

16. Muñoz (2010). Tablas de uso Práctico de los Alimentos de Mayor Consumo. México, DF: McGraw-Hill; 2010.

17. Ortega González, J. A., De Gamboa, O. F., Aintzane, A., Bilbao-Reboredo, T., Vélez-Pliego, M., \& Torres-Rasgado, E. (2017). Estado de la composición corporal del estudiante de la Facultad de Medicina de una universidad pública de México. Rev Cuban Aliment Nutr, 27(1), 14-28.

18. Pérez Ugidos, G., Lanío, F. A., Zelarayán, J., \& Márquez, S. (2014). Actividad física y hábitos de salud en estudiantes universitarios argentinos. Nutr Hosp, 30(4), 896-904. DOI:10.3305/nh.2014.30.4.7641

19. Rangel Caballero, L. G., Rojas Sánchez, L. Z., \& Gamboa Delgado, E. M. (2015). Sobrepeso y obesidad en estudiantes universitarios colombianos y su asociación con la actividad física. Nutr Hosp, 31(2), 629-636. DOI:10.3305/nh.2015.31.2.7757

20. Rizo-Baeza, M. M., González-Brauer, N. G., \& Cortés, E. (2014). Calidad de la dieta y estilos de vida en estudiantes de Ciencias de la Salud. Nutr Hosp, 29(1), 153-157. DOI:10.3305/nh.2014.29.1.6761

21. Sánchez-Socarrás, V. \& Aguilar-Martínez, A. (2015). Hábitos alimentarios y conductas relacionadas con la salud en una población universitaria. Nutr Hosp, 31(1):449-457. DOI:10.3305/nh.2015.31.1.7412

22. Savegnago Mialich, M., Covolo, N., Cheli Vettori, J., Jordao Junior, A. A. (2014). Relación entre la composición corporal y nivel de actividad física en estudiantes universitarios. Rev Chil Nutr, 41(1), 46-53.

23. Shamah-Levy, T., Cuevas-Nasu, L., Rivera-Dommarco, J., \& Hernández-Ávila, M. (2016). Encuesta Nacional de Nutrición y Salud de Medio Camino 2016 (ENSANUT MC 2016). Informe final de resultados. Disponible en: http://promocion.salud.gob.mx/dgps/descargas1/ doctos_2016/ensanut_mc_2016-310oct.pdf

24. Soto-Ruiz, M. N., Aguinaga-Ontoso, I., Canga-Armayor, N., GuillénGrima, F., Hermoso de M. J., Serrano-Monzo, I., \& Marín-Fernández, B. (2015). Modificación del peso corporal de los estudiantes universitarios en Navarra durante los tres primeros años de universidad. Nutr Hosp, 31(6), 2400-6. DOI:10.3305/nh.2015.31.6.8686

25. Suverza, A. \& Haua, K. E. El ABCD de la evaluación del estado de nutrición. México, DF: McGraw-Hill; 2010.

26. Wiklund, P. (2016). The role of physical activity and exercise in obesity and weight management: Time for critical appraisal. J Sport Health Sci, 5(2), 151-154. DOI: 10.3305/nh.2015.31.2.7757. 